\title{
Why is COPD phenotyping like sorting diamonds?
}

\author{
Peter G. Gibson ${ }^{1,2}$ and Vanessa M. McDonald ${ }^{1,2}$
}

Affiliations: 'Priority Research Centre for Asthma and Respiratory Disease, Faculty of Health and Medicine, The University of Newcastle, Newcastle, Australia. ${ }^{2}$ Dept of Respiratory and Sleep Medicine, John Hunter Hospital, Newcastle, Australia.

Correspondence: Peter G. Gibson, Priority Research Centre for Asthma and Respiratory Disease, The University of Newcastle, 1 Kookaburra Circuit, New Lambton Heights, 2305, NSW, Australia.

E-mail: peter.gibsonanewcastle.edu.au

@ERSpublications

We should work to improve the definition and recognition of asthma-COPD overlap syndrome http://ow.ly/xbFgy

The importance and management of asthma-chronic obstructive pulmonary disease overlap syndrome (ACOS) is a topic of lively debate and, until recently, there have been very few data related to this topic that could be used to inform guidelines and practice. Indeed, whether or not ACOS exists and whether it matters is central to this debate.

We can all recognise pure cases of asthma and chronic obstructive pulmonary disease (COPD) by their welldescribed clinical and pathological features. These "gems" are regularly presented as being typical of the disease, with differentiation between asthma and COPD promoted as a key goal of clinical diagnostics [1,2]. What we have been slow to accept are cases where the differentiation is not clear because of overlapping clinical features that manifest as "impurities" in the diagnosis. It now turns out that these diagnostic impurities are the rule rather than the exception.

The same can be found with diamonds. We all value a pure gem and diamond experts measure this as a property called clarity. Clarity is graded by major societies on a scale from flawless to imperfect, a bit like our peer-review system really. Only about $20 \%$ of all diamonds have a clarity grading that is high enough for the diamond to be "diagnosed" as a gemstone; the other $80 \%$ are relegated to industrial use only. Of that top $20 \%$, the harder you look, the more impurities you find.

So is the situation with airway disease. Pure cases of asthma or COPD are the minority [3] but it is this paradigm that is prized by our teachers and opinion leaders. In the older age group, the majority of cases that are seen in day-to-day clinical practice (i.e. industrial) have impure clinical features. While diamond experts have a clear and systematic approach to diagnosing impure diamonds, this is not the case in ACOS, as we have no consensus definition and, until recently, they have been neglected, and excluded from treatment trials and from guideline recommendations. This situation may be changing, thanks to studies like that of HARDIN et al. [4] who, in this issue of the European Respiratory Journal, present a characterisation of the clinical features, imaging and genetics of the "impure diamonds" of ACOS.

\section{ACOS is a global problem}

Despite the previously limited acceptance of ACOS, studies now clearly show that this is a global problem. HARDIN et al. [4] confirm ACOS in North American Caucasians and demonstrate ACOS in African Americans. ACOS has also been reported in Europeans [5, 6], in Australians and New Zealanders [3, 7-9], in Latin American populations [10], and in Asian groups [11]. ACOS is therefore a consistently recognisable

Received: May 182014 | Accepted: May 202014

Conflict of interest: Disclosures can be found alongside the online version of this article at erj.ersjournals.com 
subtype of COPD that is seen around the world, the prevalence of which is also high, with one population study reporting an overlapping phenotype in greater than $50 \%$ of people of aged 50 years [3].

Does this matter? It seems so, as patients with ACOS experience a greater disease burden than those with asthma or COPD alone. HARDIN et al. [4] demonstrate this clearly in the COPDGene study. This study represents the largest sample of people with ACOS who have been clinically characterised. It confirms the work of others in showing significantly greater quality of life impairment in ACOS. In addition, we see that the BODE (body mass index (BMI), airflow obstruction, dyspnoea and exercise capacity) score was higher in ACOS. A BODE score of 3, as seen in ACOS, is associated with a 5-year mortality of 30\% compared with a 5 -year mortality of $20 \%$ when the BODE score is less than 3 , as in the COPD-alone group. Overlap subjects also had a greater exacerbation burden. They reported an average of 1.7 times more lung attacks per year in those with ACOS, with one in three ACOS patients requiring hospitalisation or emergency department attendance for a lung attack.

This increased disease burden from ACOS seen by HARDIN et al. [4] confirms the work of others, such as the PLATINO study [10]. Given the established association between mortality and both BODE score and exacerbations, this mandates that we place a greater emphasis on the identification and management of ACOS.

\section{What can explain the increased disease burden and exacerbation risk in ACOS?}

In this study, HARDIN et al. [4] used quantitative chest computed tomography analysis to characterise the patients radiologically and they showed that there was more airway disease in patients with ACOS, with increased bronchial wall thickening and less emphysema, confirming a similar report by SIMPSON et al. [12]. This is related to the extent of gas trapping and potentially symptom impairment, but it did not explain the exacerbation risk. This was also not explained by the higher BMI in ACOS. Importantly, treatment profiles were not reported, and this would be a useful future investigation to examine any changes in inhaled corticosteroid, long-acting $\beta_{2}$-agonist and long-acting muscarinic antagonist use. It is possible that the treatment intensity was different in ACOS and this may modify clinical outcomes. More detailed mechanistic studies are warranted to identify the reason for the exacerbation risk in ACOS.

\section{The genetics of ACOS}

A clear goal of COPDGene study was to identify specific genetic markers for ACOS. None of the markers identified reached statistical significance, which confirms work of others showing that the genetics of asthma and COPD appear similar. This was identified previously when MEYERS et al. [13] reported IL13 (interleukin-13) to be a common susceptibility gene for asthma and COPD. Recently, KANEKO et al. [14] integrated the published asthma and COPD genetic studies, and found considerable overlap in genetic markers of susceptibility for asthma and COPD. A network analysis was undertaken and over 200 overlapping molecules were identified that formed a single complex network. This would suggest that there are unlikely to be specific genetic markers that will differentiate ACOS from asthma or from COPD alone.

HARDIN et al. [4] chose a different interpretation and considered the genetics underpowered, with the familiar implication that all we need is more (genome-wide association study (GWAS)) data. Certainly, there were some markers that looked interesting, even though they failed to reach statistical significance. But before rushing in with more and more GWAS data, we should pause and consider study design. With modern genetics, we obtain incredible precision where the genotype is reported to the level of individual nucleotides. Contrast this to the clinical characterisation, where ACOS is determined by answers to questions like "do you wheeze?" and "when did it begin?". Perhaps genetic epidemiologists should demand much better clinical precision, approaching that offered by genetics. Resolving this precision mismatch just might clean up this whole area. A quote from 2004 seems relevant here; it asks us to reflect on what have we done in clinical phenotyping over the last 10 years: "Now that we have sophisticated molecular level genotyping, we need to employee [sic] equally sophisticated phenotyping. Only when both are employed with equal precision, will progress be made toward linking specific genes to specific phenotypes" [15].

COPDGene does this with detailed imaging of the lung. The authors were persuaded by some of the singlenucleotide polymorphisms identified, which can be related to ACOS with biological plausibility. These relate to concepts of the origin of ACOS as a phenotype that is believed to start with asthma, which then develops into secondary COPD from associated smoking, longstanding severe asthma or other unidentified exposures. In this context, the association of ACOS with SOX5 is interesting, as this gene is associated with both COPD and abnormal lung development [16]. There is mounting evidence showing lung function defects in asthma during the period of lung growth and development in utero and in childhood [17] that persist into adult life and could lead into COPD. GPR65 was also linked to ACOS. It is associated with eosinophil activation, a typical feature of eosinophilic asthma. 
Another approach might be to identify biomarkers that are typical of ACOS. IWAMOTO et al. [18] reported elevated sputum lipocalin, a neutrophil marker, as differentiating COPD and ACOS. In contrast, BAFADHEL et al. [19] found that the mediator profile reflected the pattern of granulocytic inflammation, which is not specific for a diagnosis of asthma or COPD. This makes more biological sense when one recognises the heterogeneity of the inflammatory responses seen in airway diseases. Induced sputum eosinophilia predicts response to inhaled corticosteroid in ACOS, just as in asthma and COPD [20].

ACOS exists as a recognisable phenotype of COPD and severe asthma. It is important because the ACOS phenotype of COPD is associated with an increased illness burden that may be modifiable. Additionally, ACOS or its elements may precede the development of severe COPD, and so there is the possibility that ACOS recognition and management offers a path to COPD prevention.

\section{What needs to happen now?}

We should work to improve the definition and recognition of ACOS. Consensus meetings may do this, as the successful Ciba symposia showed in the last century. Studies like that of HARDIN et al. [4] offer the possibility of specific testing but require replication. ACOS now needs to be included in guidelines and randomised controlled trials of COPD treatment. Just as a diamond requires a multidimensional assessment that includes colour, cut, clarity and carat to determine its value and ultimate use, so in COPD, multidimensional assessment may prove a useful path to revealing the true value of what we can offer our patients [21]!

\section{References}

1 Global Initiative for Asthma. Global strategy for asthma management and prevention. www.ginasthma.org/local/ uploads/files/GINA_Report_2014.pdf

2 Global Initiative for Chronic Obstructive Lung Disease. Global Strategy for the Diagnosis, Management and Prevention of Chronic Obstructive Pulmonary Disease. www.goldcopd.org/uploads/users/files/GOLD_Report2014_ Feb07.pdf Date last updated: February 7, 2014.

3 Marsh SE, Travers J, Weatherall M, et al. Proportional classifications of COPD phenotypes. Thorax 2008; 63: 761-767.

4 Hardin M, Cho M, McDonald M-L, et al. The clinical and genetic features of COPD-asthma overlap syndrome. Eur Respir J 2014; 44: 341-350.

5 de Marco R, Pesce G, Marcon A, et al. The coexistence of asthma and chronic obstructive pulmonary disease (COPD): prevalence and risk factors in young, middle-aged and elderly people from the general population. PLoS One 2013; 8: e62985.

6 Andersen $\mathrm{H}$, Lampela P, Nevanlinna A, et al. High hospital burden in overlap syndrome of asthma and COPD. Clin Respir J 2013; 7: 342-346.

7 McDonald VM, Simpson JL, Higgins I, et al. Multidimensional assessment of older people with asthma and COPD: clinical management and health status. Age Ageing 2011; 40: 42-49.

8 Fu JJ, Gibson PG, Simpson JL, et al. Longitudinal changes in clinical outcomes in older patients with asthma, COPD and asthma-COPD overlap syndrome. Respiration 2014; 87: 63-74.

9 Weatherall M, Travers J, Shirtcliffe PM, et al. Distinct clinical phenotypes of airways disease defined by cluster analysis. Eur Respir J 2009; 34: 812-818.

10 Menezes AM, Montes de Oca M, Perez-Padilla R, et al. Increased risk of exacerbation and hospitalization in subjects with an overlap phenotype: COPD-asthma. Chest 2014; 145: 297-304.

11 Rhee CK, Yoon HK, Yoo KH, et al. Medical utilization and cost in patients with overlap syndrome of chronic obstructive pulmonary disease and asthma. COPD 2014; 11: 163-170.

12 Simpson JL, Milne DG, Gibson PG. Neutrophilic asthma has different radiographic features to COPD and smokers. Respir Med 2009; 103: 881-887.

13 Meyers DA, Larj MJ, Lange L. Genetics of asthma and COPD. Similar results for different phenotypes. Chest 2004; 126: Suppl., 105S-110S.

14 Kaneko Y, Yatagai Y, Yamada H, et al. The search for common pathways underlying asthma and COPD. Int J Chron Obstruct Pulmon Dis 2013; 8: 65-78.

15 Cody JD, Hale DE. Precision in phenotyping and genotyping. Am J Med Genet A 2004; 131: 313.

16 Hersh CP, Silverman EK, Gascon J, et al. SOX5 is a candidate gene for chronic obstructive pulmonary disease susceptibility and is necessary for lung development. Am J Respir Crit Care Med 2011; 183: 1482-1489.

17 Tai A, Tran H, Roberts M, et al. The association between childhood asthma and adult chronic obstructive pulmonary disease. Thorax 2014 [In press DOI: 10.1136/thoraxjnl-2013-204815].

18 Iwamoto H, Gao J, Koskela J, et al. Differences in plasma and sputum biomarkers between COPD and COPDasthma overlap. Eur Respir J 2014; 43: 421-429.

19 Bafadhel M, McCormick M, Saha S, et al. Profiling of sputum inflammatory mediators in asthma and chronic obstructive pulmonary disease. Respiration 2012; 83: 36-44.

20 Kitaguchi Y, Komatsu Y, Fujimoto K, et al. Sputum eosinophilia can predict responsiveness to inhaled corticosteroid treatment in patients with overlap syndrome of COPD and asthma. Int J Chron Obstruct Pulmon Dis 2012; 7: 283-289.

21 McDonald VM, Higgins I, Wood LG, et al. Multidimensional assessment and tailored interventions for COPD: respiratory utopia or common sense? Thorax 2013; 68: 691-694. 